\title{
Physically-Based Interaction for Networked Virtual Environments
}

\author{
Christoph Anthes, Roland Landertshamer, and Jens Volkert \\ GUP, Institute of Graphics and Parallel Processing \\ Johannes Kepler University, Altenbergerstrasse 69, A-4040 Linz, Austria \\ canthes@gup.uni-linz.ac .at
}

\begin{abstract}
Physics engines are gaining importance in the area of computer games to create better visual experiences. In the context of networked Virtual Reality (VR) applications they can be useful to provide new interaction possibilities. This paper will provide an introduction into the area of physics simulation and the use of it to create more realistic and interactive multi-user virtual worlds. Examples for the usefulness of such physics engines can be found in a variety of training applications.
\end{abstract}

\section{Introduction}

In the past years Virtual Reality (VR) has become a useful tool for a variety of application areas. Networked Virtual Environments (NVEs) allow dislocated users from all over the globe to communicate and interact in a shared virtual space.

If we take a look at safety training applications like the SAVE project [12 where the user has to react on hazardous incidents in a petroleum refinery or the VETT project, which provides shipboard firefighting training [3] we discover that most of them are single user applications or work with two users a trainer and a single trainee, where only the trainee enters the VE and gets instructions from the trainer. In the real world safety training is typically performed in teams, as hazardous incidents are fought by teams. Due to that reason it is obvious that safety training VR applications would profit from multi-user interaction and collaboration.

Another issue which arises is the fidelity of interaction. Users are typically interacting with a Virtual Hand interaction technique. If the representation of the users cursor collides with an entity in the VE interaction can be performed. The level of interaction is normally reduced to clicking on the entity which can for example result in the triggering of an event. This is sufficient to train basic procedures during incidents. To maximise the training in such environments physics engines should be incorporated to allow for a more realistic behaviour of the $\mathrm{VE}$, where the user has to perform the movement of the object manipulation like he would in the real world. This could be throwing a lever, or pushing down a virtual door handle in order to pull the door open afterwards. 
The in VRs framework 11 is designed to ease the creation of NVEs. Its physics module is used to implement realistic interaction possibilities with the environment. Joints for example can interconnect nodes of the scene graph and allow for a simple scene description. Gravity and collision detection become important during manipulation and placement tasks. This makes in VRs an ideal candidate to create NVEs for safety training.

This Section has given a brief introduction on the use of physics in training environments. The next Section will provide an overview on the related work. In the following the use of physics will be described in the context of the framework. Network communication for the physically based interaction will be introduced and some example setups will be given. The final Section concludes the paper and gives an outlook of future developments.

\section{Related Work}

Problem domain and related work of this paper are distributed over three different areas, physics, network communication and interaction.

A detailed overview on the design of a $3 \mathrm{D}$ rigid body physics engine for games is given by David H. Eberly [4. He describes in detail the mathematical and physical background while Baraff focuses in his thesis [5] on how physical constraints can be transformed in to mathematical problems and how they can be solved.

The commonly available NVEs like DIVE [6, AVOCADO [7] or MASSIVE [8], do not support the use of physics engines due to several reasons. Simulating physics in none client-server topologies is very cumbersome. In a peer-to-peer (p2p) topology one client would have to be selected to perform the role of a server, to guarantee a consistent state of the VE. Most of the existing VR systems incorporate $\mathrm{p} 2 \mathrm{p}$ topologies or hybrid approaches, to achieve a high responsiveness and scalability. Another issue which constraints the use of physics is the computational intensity of such simulations. A good overview on NVEs in general is given by Singhal and Zyda in [9].

A variety of interaction techniques for VEs has been described by Mine. 10]. The psychological aspects of collaborative object manipulation have been researched on many levels by Roberts et al. [11. On the technical level Broll gives ideas on how to solve concurrent object manipulation [12].

Jorisson and Lamotte combine the use of physics engines and the VR to create a platform for collaborative VEs where objects contain the interaction information and behaviour [13. Their networking topology uses a classic client server approach, where one server is responsible for the physics update of the whole VE.

Early approaches in scene graphs like VRML and Inventor use sensors to constrain object movement. The use of joints provides similar functionality but provides additional features like the swinging of a door.

$\overline{1 \text { Pronounced }}$ in'v3:s. 


\section{$3 \quad$ Framework Architecture}

The inVRs framework consists of modules for navigation, interaction and network communication. These modules are interconnected via a system core, which provides databases to store information about the VE and the users inside the $\mathrm{VE}$ as well managers for communication handling between the modules and the databases. A brief overview on the architecture is given by Anthes and Volkert [14. in VRs provides the possibility to concurrently manipulate the same geometry as the same time by two dislocated users. It supports this collaboration in many ways including the use of an additional physics module.

One of the important aspects of the framework is the distinction between discrete events which are distributed via the event manager and continuous streams of transformation data which is handled by the transformation manager. A detailed description of the transformation and event handling can be found in [15. The physics module makes use of these two managers, to generate for example collision events or change the transformations of the objects in the NVE.

\section{Physics Module}

To increase the immersion of the users in a VE it is important that the virtual objects show a realistic behaviour. Therefore a physics engine can be used. In the context of VEs it is responsible for the simulation of the behaviour of rigid bodies, which comprises the following tasks:

- rigid body motion

- collision detection and response

- constrained interaction of rigid bodies

Each object in the VE that should be simulated by the physics engine has to be represented by a physics object. For rigid body motion this physics object has to contain the mass and the inertia tensor of the virtual object. With this information the physics simulation can calculate the linear and angular velocities of the object and determine its position and orientation. The resulting velocities of the objects are a result of forces and torques which act on the physics object. To avoid that two physics objects interpenetrate the physics engine has to check for collisions. Therefore each physics object needs a description of its shape. In general the triangle mesh of the virtual object could be used for the collision detection but in real time simulations the shape of the objects is often approximated by 3D primitives like spheres or boxes to reduce the amount of computation time. When the physics engine encounters a collision then the collision response has to be applied. In this step the physics engine calculates an impulse as a result of the collision. This impulse is then applied to both objects to avoid an interpenetration. For constrained interaction of rigid bodies the physics engines provide constraints or joints. A joint describes a restriction of motion for a physically simulated object. Joints can be used to connect two objects together and/or allow only special relative movement between two objects. A joint can 
also be used to restrict the motion of a single object. The possibilities of motion restriction cover the free rotation around a point, the rotation around an axis, the movement along an axis or any combination of this constraints. Each joint can again be restricted in it's movement range. This allows to define a rotation to a maximum angle or the movement to a maximum distance.

The physics module of the in VRs framework uses a physics engine to allow constrained interaction with virtual objects. It therefore allows to connect virtual objects via joints. XML is used to define the properties of the joints and to interconnect nodes of the scene graph. Since each entity in the VE has a unique id, which is stored in a database of the framework it is possible to interconnect entities based on their ids. The physics module allows to define thresholds for each joint to execute events when these thresholds are exceeded. The possible types of thresholds depend on the types of the joint. Examples are a rotation angle around the joint axis or a distance from the untransformed position. The events that should be executed can be defined in the config file. For each joint the user can set activation conditions which describe when the joint should be active and when not. An inactive joint is seen as a fixed connection between two objects. The physics module of the in VRs framework allows to activate or deactivate a joint when a user interacts with an entity, when other joints are active or not, when a joint exceeds a defined angle or a joint exceeds a predefined distance. An example for this conditions can be found in the Section 6 .

\section{Network Communication}

To synchronise VEs typically locking mechanisms are used. The philosophy of the in VRs framework is to avoid locking and keeping a loose consistency. The presented approach transmits a continuous stream of transformation data packets via UPD. The transformation data is mostly generated by the tracking systems of the interconnected VR installations. Transformations from objects in the scene can be transmitted as well if necessary via this distribution channel. The network topology of the framework is a peer-to-peer topology, which works with fully replicated databases of the NVE to guarantee a high responsiveness.

Since tracking information can be considered ubiquitous if two or more users are interacting in an NVE very little additional messages are needed to implement physically based interaction or concurrent object manipulation. If a local user picks a part of a physically simulated entity, which could be for example a door handle or a vent in a VE, a pick event is sent to the remote users. This message is transfered in a reliable way and consists of a unique user and entity id. Additionally it contains information about the time, when the entity was picked and an offset from the origin of the entity to the picking point, which is determined based on the interaction technique of the user (e.g. ray-casting or virtual hand). The remote systems are now notified that the user is connected with the entity. With the information about the offset to the users picking point and the additional tracking data they are able to perform a local physics simulation. 
Once the object is released a reliable message is sent which stores data about the exact position where the object is dropped with an additional timestamp. The remote clients interpolate the position and orientation of the object between current transformation at the time they received the message to the transformation in the time in the past which is stated in the timestamp of the release message. The duration of this interpolation can be set in system setting of the framework.

Using this type of synchronisation, object manipulation can be performed locally without the need of additional transformations for each simulated object. By using reliable release messages and transforming the object to a final position the NVE is brought back into a consistent state.

\section{Example Setups}

A variety of application areas of physics in a VE arise. An example for the use of joints would be a door. The same mechanisms could be applied on any entities in the VE. They are ideal to implement vents or handles in a safety training application. Furthermore object manipulation with a virtual hand technique or concurrent object manipulation can be handled by the use of physics.

\subsection{A Door in the VE}

In this example the scene graph for the door consists of three interconnected nodes. The frame, the actual door and a door handle. The the door is attached to the frame with a hinge joint on the z-axis. The rotation around the frame is restricted to +/- 90 degrees. Additionally a threshold area of an angle of 1 degree is defined in which the door is considered closed. If no user is interaction with the door and it is in that threshold area it will be rotated to it's idle state and will be deactivated. The handle is as well attached to the door with a hinge joint. It rotational freedom is limited 45 degrees around the y-axis. Thresholds measure when it is rotated more than 25 degrees. In this case the joint between the frame and the door is activated and the door can be rotated. Figure 1 illustrates the setup of such a door while Listing 1.1 shows the XML definition of the example joint setup.

In the initial state frame, door and handle are in passive mode. None of these joints are simulated by the physics module at this stage. The user can grab the handle, if this action has taken place, the joint between handle and door is activated and simulated by the physics module. Once the user has rotated the handle over the threshold, the joint between the door and the frame become active. The whole door is now simulated in the physics module. since the axes of the two joints are independent no problems can arise during the calculation of the position of the door and it's parts, it is always unique.

Using these joints for interaction does not only provide easy mechanisms to create functional elements in a VE. Furthermore it give the VE a very realistic 
feeling, when the user has the possibility to swing doors. It still has to be proven, that by actually pulling down a door handle and pulling the door open the user is trained in a better way than he is by simply clicking on the object.
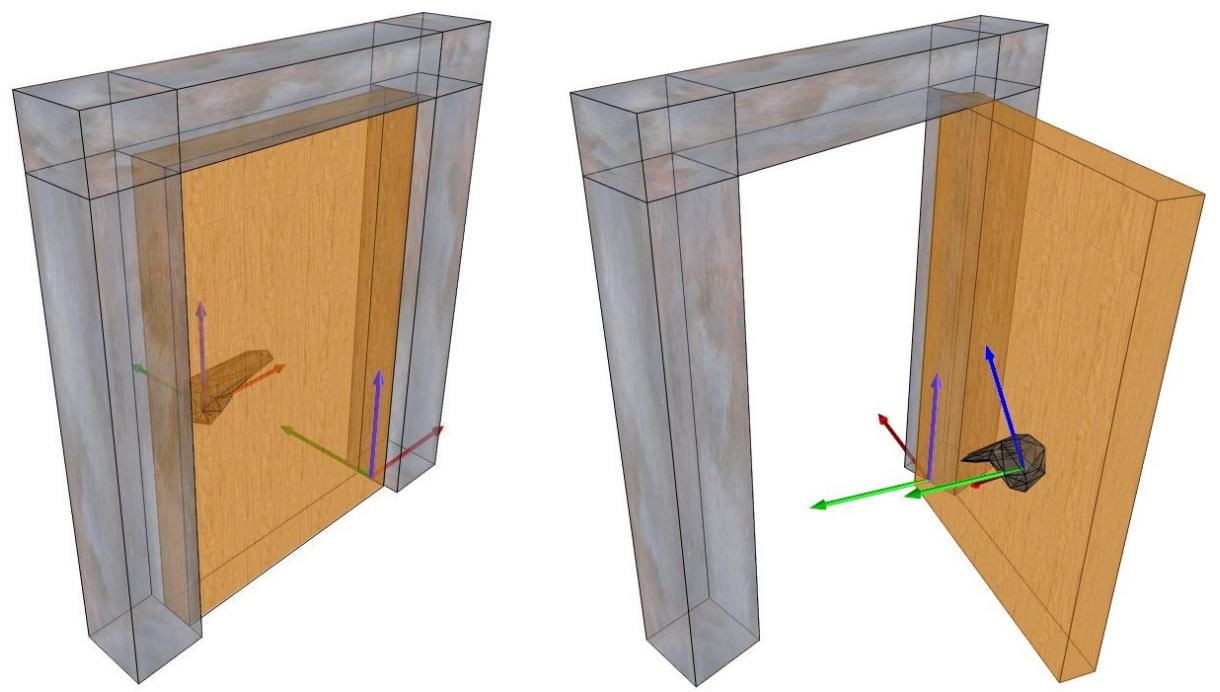

Fig. 1. A door in the VE

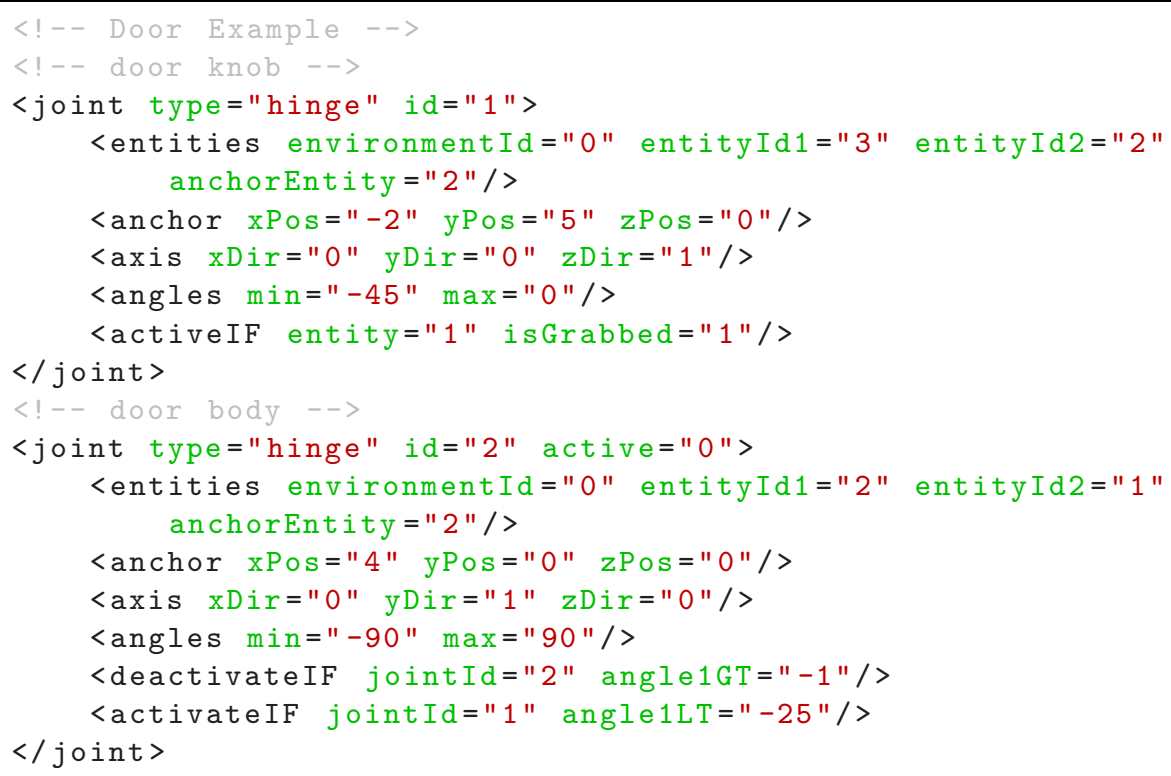

Listing 1.1. Joint definition of a door 


\subsection{Natural Interaction}

To realise natural interaction tracking systems are incorporated. The movement of the users input device is directly mapped on the cursor position and orientation in the VE. The physics module can be used to simulate object properties like gravity.

If gravity is used in the physics simulation of the environment one client has to act as a master for the simulation. When an object is dropped gravity has to be calculated and the proper rebound has to be calculated. This type of simulation has to be calculated locally by the master client, who is in controll of the object. The transformations of the matrices have to be transferred over the network.

\subsection{Concurrent Object Manipulation}

Concurrent object manipulation as described by Broll [12] allows two users to manipulate the same attribute of the same virtual object in real-time. This type of interaction can be extremely useful in construction scenarios or safety applications. Obstacles could be carried away or building material could be arranged.

The VR systems mentioned in Section 2 do not support cooperative manipulation. They lock the access to an object to a exclusively to a single user. Broll suggests to combine interaction requests and calculate the resulting object position on one participants site, to keep the system consistent. An alternative approach was developed by Froehlich et al. [16] who incorporate physics to cooperatively manipulate objects during assembly tasks. The approach developed by Froehlich attaches a physically simulated spring between the cursor of the user and the point, where the user grabbed the object.

In our case concurrent object manipulation is detected if two transformation on the same object are detected by the transformation manager. In that case a special merging is introduced which can be implemented using Froehlich's physics approach. The resulting transformation is applied on the object. Since the immediate input transformations from the local user and slightly delayed transformations from the remote user which still can be extrapolated are available it is possible to provide a relatively correct and highly responsive representation of the cooperatively manipulated object.

\section{Conclusions and Future Work}

This paper has given an introduction on the use of physics simulation for interaction in NVEs. A physics module for the the inVRs framework allows to define joints for interconnection of scene graph nodes. These nodes can be used for highly interactive NVEs. Three types of interaction have demonstrated the use of physics simulation for VEs, especially training scenarios.

Advanced methods for synchronsiation of physics have to be found. A similar approach than synchronising particle systems on multiple displays might be used. The distribution of random seeds for some aspects of the physics calculation might help to simulate parts of the VE locally. 


\section{References}

1. Haller, M., Holm, R., Volkert, J., Wagner, R.: A vr based safety training in a petroleum refinery. In: Annual Conference of the European Association for Computer Graphics (EUROGRAPHICS '99). (1999)

2. Haller, M., Kurka, G., Volkert, J., Wagner, R.: omvr - a safety training system for a virtual refinery. In: ISMCR '99, Tokyo, Japan (1999)

3. Tate, D.L., Sibert, L., King, T.: Virtual environments for shipboard firefighting training. In: IEEE Virtual Reality Annual International Symposium (VRAIS '97), Albuquerque, NM, USA, IEEE Computer Society (1997) 61-68

4. Eberly, D.H.: Game Physics. The Morgan Kaufmann Series in Interactive 3D Technology. Morgan Kaufmann (2004)

5. Baraff, D.: Dynamic Simulation of Non-Penetrating Rigid Bodies. PhD thesis, Department of Computer Science, Cornell University, Ithaca, NY 14853-7501, USA (1992)

6. Carlsson, C., Hagsand, O.: Dive - a multi-user virtual reality system. In: IEEE Virtual Reality Annual International Symposium (VRAIS '93), Seattle, WA, USA, IEEE Computer Society (1993) 394-400

7. Tramberend, H.: Avocado: A Distributed Virtual Environment Framework. PhD thesis, Technische Fakultät, Universität Bielefeld (2003)

8. Greenhalgh, C., Benford, S.: Massive: A distributed virtual reality system incorporating spatial trading. In: IEEE International Conference on Distributed Computing Systems (DCS '95), Vancouver, Canada, IEEE Computer Society (1995) $27-34$

9. Singhal, S.K., Zyda, M.J.: Networked Virtual Environments - Design and Implementation. Addison-Wesley Professional (1999)

10. Mine, M.R.: Virtual environment interaction techniques. Tr95-018, University of North Carolina, Chapel Hill, NC 27599-3175 (1995)

11. Roberts, D.J., Wolff, R., Otto, O., Steed, A.: Constructing a gazebo: Supporting teamwork in a tightly coupled, distributedtask in virtual reality. Presence: Teleoperators and Virtual Environments 12 (2003) 644-657

12. Broll, W.: Interacting in distributed collaborative virtual environments. In: IEEE Virtual Reality Annual International Symposium (VRAIS '95), Los Alamitos, CA, USA, IEEE Computer Society (1995) 148-155

13. Jorissen, P., Lamotte, W.: Dynamic physical interaction platform for collaborative virtual environments. In: CollabTech 2005, Tokyo, Japan (2005)

14. Anthes, C., Volkert, J.: invrs - a framework for building interactive networked virtual reality systems. In: International Conference on High Performance Computing and Communications (HPCC '06), Munich, Germany, Springer (2006) 894-904

15. Anthes, C., Landertshamer, R., Bressler, H., Volkert, J.: Managing transformations and events in networked virtual environments. In: International MultiMedia Modeling Conference (MMM '07), Singapore, Springer (2007)

16. Fröhlich, B., Tramberend, H., Beers, A., Agrawala, M., Baraff, D.: Physically-based manipulation on the responsive workbench. In: IEEE Virtual Reality (VR '00), New Brunswick, NJ, USA, IEEE Computer Society (2000) 5-12 